\title{
THz Modulators and Detectors Based on Semiconductor Nanowires
}

\author{
Michael B Johnston \\ Department of Physics, University of Oxford, \\ Clarendon Laboratory, Parks Road Oxford, OX1 3PU, United Kingdom
}

\begin{abstract}
Semiconductors nanowires have to potential to be building blocks for future nano-optoelectronic devices. We have recently demonstrated high performance $\mathrm{THz}$ photonic devices based on GaAs and InP nanowires. These include ultrafast optically switched modulators of $\mathrm{THz}$ radiation and single nanowire photoconductive detectors of $\mathrm{THz}$ pulses.
\end{abstract}

\section{INTRODUCTION}

$\mathrm{T}$ erahertz technologies and applications have developed rapidly over the past few decades, however applicatuions in areas such as high-speed communications are limited by a lack of some components such as very high-speed modulators.[1] We have been expoliting the unique properties of semiconductor nanowires to develop new $\mathrm{THz}$ devices including ultrfast $\mathrm{THz}$ modulators and detectors.

Semiconductor nanowires of group III-V semiconductors can be grown as defect-free single crystals by molecular beam epitaxy (MBE) or metal-organic vapour phase epitaxy (MOVPE) [2], and hence they possess excellent electronic properties, such as very high electron mobilities, and may be doped precisely [3]. In addition the $\sim 10 \mathrm{~nm}$ diameters of the nanowires allow unique structures to be formed, such as heterojunctions with lattice mismatched materials, and both axial and radial quatum heterostructures [4].

We have previously developed techniques to characterise and optimise semoconductor nanowires based on optical pump terahertz probe spectroscopy [5]. Based on this work we have now developed nanowires and nanowire devices specifically for applications on $\mathrm{THz}$ science and technology.

\section{AN UltRAFAST THZ MODULATOR}

GaAs semiconductor of diameter $50 \mathrm{~nm}$ were grown by MOVPE at the Australian National University using Auassisted Vapour-Liquid-Solid metthod [2] (as shown in Fig $1 b)$. The nanowires were transferred onto parylene-C films such that the nanowires were arranged in a grid fashion. [6] Layers of parylene with nanowires embedded were laminated to form a flexible active polariser element, as example of which is shown in Fig. 1c. The concept of the $\mathrm{THz}$ modulator is shown in Fig 1a. The parylene-nanowire film acts as a wire-grid $\mathrm{THz}$ polariser when optically excited but just acts as a dielectric slab when not photoexcited. As the photoconductivity lifetime is very short in GaAs nanowires, the device can be switched on a picosecond timesacle. We previuosly demonstrated this concept with semoconducting carbon nanotubes [7], but he modulation depth was poor.

We utilised an optical pump-terahertz probe spectroscopy system [5] and varied the polarization of the optical pump beam to demonstrate the ultrafast parylene-nanowire $\mathrm{THz}$ modulator. The modulatuion switching time was found to be less than $5 p$ and a modulation depth of $-8 \mathrm{~dB}$. We achieved an extinction of over $13 \%$ and $4 \mathrm{THz}$ bandwidth.

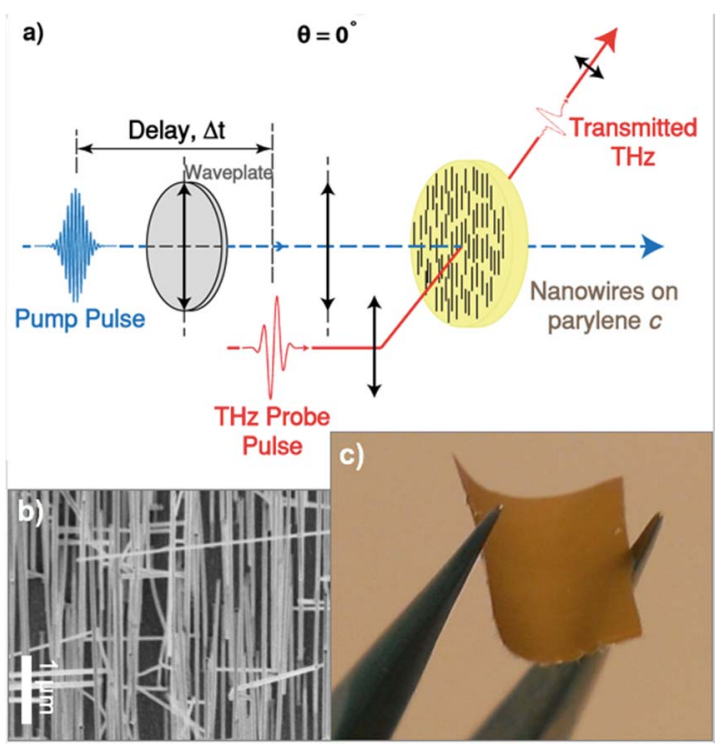

Fig. 1. (a) Schematic diagram showing the concept and experimental set-up of the ultrafast $\mathrm{THz}$ modulator. (b) SEM image of GaAs nanowires grown on the GaAs substrate before embedding in parylene (c) Photograph of flexible modulator film consisting of aligned GaAs nanowires embedded in parylene.

\section{SINGLE NANOWIRE THZ DETECTORS}

We have also recently used selective n-type doping along InP nanowires to improve the design and performance of our single nanowire photoconductive $\mathrm{THz}$ receivers $[7,8]$.

\section{REFERENCES}

[1] SS Dhillon, et al. The 2017 terahertz science and technology roadmap. Journal of Physics D: Applied Physics 50 (4), 043001 (2017)

[2] HJ Joyce, et al "III-V semiconductor nanowires for optoelectronic device applications" Prog. Quantum Electron., 35:23-75 (2011)

[3] JL Boland et al, "Increased Photoconductivity Lifetime in GaAs Nanowires by Controlled n-Type and p-Type Doping" ACS Nano, 10, 42194227 (2016)

[4] Davies et al. Low Ensemble Disorder in Quantum Well Tube Nanowires Nanoscale, 7, 20531 (2015)

[5] HJ Joyce, JL Boland, CL Davies, SA Baig, MB Johnston, "A review of the electrical properties of semiconductor nanowires" Semicond. Sci. and Technol. 31 (10), 103003 (2016)

[6] SA Baig, JL Boland, DA Damry, HH Tan, C Jagadish, HJ Joyce, MB Johnston. "An ultrafast switchable terahertz polarization modulator based on III-V semiconductor nanowires". Nano Letters (in press)

[7] Docherty et al., An ultrafast carbon nanotube terahertz polarisation modulator. J. Appl. Phys., 20, 115 (2014)

[8] K Peng, et al. Single $\mathrm{n}^{+}-\mathrm{i}-\mathrm{n}^{+}$InP nanowires for highly sensitive terahertz detection. Nanotechnology 28 (12), 125202 (2017)

[9] K Peng et al, Broadband Phase-Sensitive Single InP Nanowire

Photoconductive Terahertz Detectors. Nano Letters 16 (8), 4925-4931

\section{ACKNOWLEDGEMENT}

The EPSRC (UK) and ARC (Australia) are gratefully acknowledged for financial support. This was collaborative work with the Jagadish group (ANU) and Joyce group (Univ. Cambridge). 